onomy. The chairman of the Subgroup on Taxonomy of Toxoplasma presented a progress report.

Minute 6. Acceptance of a standard antitoxoplasma serum. After an international assay had been carried out under the auspices of an Expert WHO Committee, a standard antitoxoplasma serum was accepted by the Biological Standardization Committee. This serum is supplied on demand from the Department of Standardization at the State Serum Institute in Copenhagen. The establishment of reference laboratories in different geographic areas was recommended but has not yet been implemented.

Minute 7. Future work. Future work will continue the attempts to define the biological relationships of the toxoplasmas.

Minute 8. Next meeting. The next meeting of this subcommittee will be held in conjunction with the next International Congress.

Minute 9. Open meeting. An open meeting of the subcommittee was held on 11 August 1970.

Minute 10. Present membership. The present membership of the subcommittee is as follows: J. Chr. Siim (Chairman), Copenhagen, Denmark; C. R. Cole, Columbus, Ohio, USA; H. A. Feldman, Syracuse, New York, USA; P. C. C. Garnham, Berkshire, England; D. N. Zasukhin, Moscow, USSR.

Minute 11. Adjournment. The meeting was adjourned.

\title{
Report (1969-1970) of the Subgroup on Taxonomy of Toxoplasma of the Subcommittee on the Genus Toxoplasma
}

\author{
14 August 1970
}

Mexico City, Mexico

Since the last informal meeting of the subcommittee in Leningrad in July 1969, the problem of the classification of Toxoplasma has become clearer because of new discoveries concerning the life cycle of the organism.

The research began when Hutchison showed in 1965 that the feces of cats, which had been fed cysts of Toxoplasma gondii, contained a resistant, long-lived, infectious form of the organism.

It was finally proved late in 1969 that the nature of this form was coccidian, first by Siim, Hutchison, and Work, and almost simultaneously by Frenkel and Dubey, and by Sheffield. The work was further confirmed by observers in Germany (Piekarski, Werner, Janitschke, and Weiland), in Holland (Overdulve), and in Hawaii (Wallace).

Oocysts seen in the cat's feces possessed a disporocystic, tetrazoic structure, and the endogenous stages were those of a typical coccidian with rapid schizogony and gametogony throughout the small intestine.

Some workers at once jumped to the conclusion that the genus Toxoplasma would have to be replaced by the genus Isospora, owing to the structure of the oocyst, and this was attempted in a publication by Overdulve (1970). It was a "conditional proposal," not in accordance with the new "Règles." However, the similarity in the structure of the oocyst does not necessarily mean that the genera are the same. For example, there are several compartments in Hoare's table of the coccidia which contain more than one genus (e.g., Pfeifferinella, Schellackia, Tyzzeria). The profound differences in the life cycle of Toxoplasma (with its extra or aberrant cystic and pseudocystic development, involving multiplication by endodyogeny) clearly indicate that it is not Isospora, though when the full life cycle of certain species of the latter are known (e.g., $I$. hominis and $I$. bigemina), it may be necessary to remove them to Toxoplasma.

At present, therefore, it would seem unwise to interfere with the present name of Toxoplasma. It is necessary, however, to remove this genus from the subclass Toxoplasmatea, which was created for it by Biocca, and place it in the Coccidia. Scholtyseck et al. have proposed the creation of a new family, Endodyococcidia. The positions of the other genera in the subclass Toxoplasmatea-Sarcocystis, Frenkelia, and Besnoitia remain obscure at present, and it would probably be better to defer the introduction of new names until further investigations are completed on the whole group.

P. C. C. Garnham 